\title{
recillunds
}

Revista Científica Mundo de la Investigación y el Conocimiento

Luis Carlos Sanchez Lopez a ; Paola Alexandra Bonifaz Aquino ${ }^{\text {b }}$; Maria Monserrate Ponce Ventura ${ }^{c}$; Marcelo Kevin Taiano Espinoza ${ }^{d}$

Contradicciones de analgesia epidural post cesárea: ¿solución o problema?

Post-caesarean epidural analgesia contradictions: solution or problem?

Revista Científica Mundo de la Investigación y el Conocimiento. Vol. 3 núm.3. Esp., noviembre, ISSN: 2588-073X, 2019, pp. 834-852

DOI: $10.26820 /$ recimundo/3.(3.Esp).noviembre.2019.834-852

URL: http://recimundo.com/index.php/es/article/view/628

Código UNESCO: 3205 Medicina Interna

Tipo de Investigación: Artículo de Revisión

(C) RECIMUNDO; Editorial Saberes del Conocimiento, 2019

Recibido: 15/09/2019 Aceptado: 23/10/2019 Publicado: 30/11/2019

Correspondencia: medluisanchezlopez@gmail.com

\footnotetext{
a. Médico; Investigador Independiente; Guayaquil, Ecuador; medluisanchezlopez@ gmail.com

b. Médico; Investigadora Independiente; Guayaquil, Ecuador; jpau_4629@hotmail.com

c. Licenciada en Terapia Física; Médico; Tecnología en Terapia Física y Rehabilitación; Investigadora Independiente; Guayaquil, Ecuador; maia_pv21@hotmail.com

d. Médico; Investigador Independiente; Guayaquil, Ecuador; marcellotaianoespinoza@gmail.com
} 


\section{Contradicciones de analgesia epidural post cesárea: ¿solución o problema?}

Vol. 3, núm. 3 Esp., (2019)

Luis Carlos Sanchez Lopez; Paola Alexandra Bonifaz Aquino; Maria Monserrate Ponce Ventura;

Marcelo Kevin Taiano Espinoza

\section{RESUMEN}

El dolor postoperatorio es una combinación de dos procesos fundamentales, la transmisión de impulsos nocioceptivos aferentes, generados por la lesión tisular y la sensibilización central producida por un estímulo persistente. Los opiáceos medulares pueden alterar ambos procesos mediante la inhibición pre y post sináptica de la excitación neural, reduciendo el estímulo que inicia el proceso de hipersensibilización central. Es necesario que el alivio del dolor sea seguro y efectivo, que no interfiera con la capacidad de la madre para desenvolverse y realizar todo lo concerniente al cuidado de su bebé, y que no produzca efectos adversos en la lactancia. Los opioides se pueden clasificar según su lipofilicidad. Los opioides más lipofílicos como el sufentanil y el fentanil, optimizan en gran medida la calidad de la anestesia intra operatoria, tienen un inicio de acción más rápido pero una duración más corta, que los que tienen menor lipofilicidad. Los opioides hidrofílicos como la morfina tienen una duración mayor, entre 14 y 36 horas, pero con un inicio de acción más tardío, en comparación con los opioides que tienen mayor lipofilicidad. La analgesia multimodal permite conseguir una analgesia de calidad y con menos efectos secundarios gracias al uso de diferentes analgésicos o técnicas analgésicas. El uso de analgesia multimodal es importante para optimizar la calidad de la analgesia postoperatoria y disminuir así el requerimiento de opioides en el periodo postparto. Contraindicaciones: las pacientes con lesiones cardiacas obstructivas serias y compromiso hemodinámico, tales como: estenosis aórtica, estenosis mitral, hipertensión pulmonar y coartación aórtica, no son candidatas para la aplicación de ésta técnica por no tolerar los cambios rápidos a nivel de la pre y post carga. La metodología usada es descriptiva, con un enfoque documental, es decir, revisar fuentes disponibles en la red, como google académico, con contenido oportuno y relevante desde el punto de vista científico que enriquezca el análisis del tema planteado en este artículo.

Palabras claves: Nivel de dolor; Analgesia; Epidural; Opioides; No opioides; Analgesia multimodal; Post cesárea; Morfina. 


\section{Contradicciones de analgesia epidural post cesárea: ¿solución o problema?}

Vol. 3, núm. 3 Esp., (2019)

Luis Carlos Sanchez Lopez; Paola Alexandra Bonifaz Aquino; Maria Monserrate Ponce Ventura; Marcelo Kevin Taiano Espinoza

\section{ABSTRACT}

Postoperative pain is a combination of two fundamental processes, the transmission of afferent nocioceptive impulses, generated by tissue injury and the central sensitization produced by a persistent stimulus. The medullary opiates can alter both processes by pre and post synaptic inhibition of neural excitation, reducing the stimulus that starts the central hypersensitization process. It is necessary that pain relief be safe and effective, that it does not interfere with the mother's ability to function and do everything concerning the care of her baby, and that it does not produce adverse effects on breastfeeding. Opioids can be classified according to their lipophilicity. The more lipophilic opioids such as sufentanil and fentanyl, greatly optimize the quality of intraoperative anesthesia, have a faster onset of action but a shorter duration, than those with less lipophilicity. Hydrophilic opioids such as morphine have a longer duration, between 14 and 36 hours, but with a later onset of action, compared to opioids that have greater lipophilicity. Multimodal analgesia allows to achieve quality analgesia and with less side effects thanks to the use of different analgesics or analgesic techniques. The use of multimodal analgesia is important to optimize the quality of postoperative analgesia and thus reduce the opioid requirement in the postpartum period. Contraindications: Patients with serious obstructive cardiac lesions and hemodynamic compromise, such as: aortic stenosis, mitral stenosis, pulmonary hypertension and aortic coarctation, are not candidates for the application of this technique for not tolerating rapid changes at the pre and post levels load. The methodology used is descriptive, with a documentary approach that is, reviewing sources available on the web, such as google scholar, with timely and scientifically relevant content that enriches the analysis of the topic raised in this article.

Keywords: Pain level; Analgesia; Epidural; Opioids; Non-opioids; Multimodal analgesia; Post cesarean section; Morphine. 


\section{Contradicciones de analgesia epidural post cesárea: ¿solución o problema?}

Vol. 3, núm. 3 Esp., (2019)

Luis Carlos Sanchez Lopez; Paola Alexandra Bonifaz Aquino; Maria Monserrate Ponce Ventura;

Marcelo Kevin Taiano Espinoza

\section{Introducción}

El control del dolor postoperatorio en pacientes sometidas a cesárea facilita una buena y más rápida recuperación y puede disminuir los costes de hospitalización. Se ha tratado de implementar una técnica analgésica que ocasione mínimos efectos secundarios pero que provea de una buena calidad y duración de la analgesia. El manejo del dolor en este tipo de pacientes está regido por varios factores adicionales: la experiencia de una cirugía previa, ansiedad, cambios emocionales, las expectativas de la madre ante el nuevo nacimiento, el umbral al dolor, la edad, etc. (Illodo, 2011).

La anestesia regional es la técnica que se prefiere sobre la anestesia general, siendo utilizada en aproximadamente un $80 \%$ de las cesáreas (Templos, 2008). Entre sus ventajas incluye la menor exposición de fármacos al neonato, disminución en el riesgo de broncoaspiración, que la madre tenga contacto inmediato con el producto al nacimiento y la opción de utilizar opioides espinales en el postoperatorio.

Existe gran debate en la literatura especializada del tema referida a si es positiva o negativa la prescripción de fármacos a pacientes post cesáreas. Todo depende del nivel del dolor de la paciente y en encontrar una fórmula que ocasiones los menores efectos negativos tanto a la madre como al neonato. El objetivo primordial del manejo racional de los fármacos reside en optimizar la eficacia y minimizar la toxicidad. La analgesia unimodal es insuficiente, por lo que se recomiendan actualmente las terapias multimodales, en las que se combinan diferentes vías de administración y diferentes fármacos analgésicos o anestésicos, lo cual produce un efecto 


\section{Contradicciones de analgesia epidural post cesárea: ¿solución o problema?}

Vol. 3, núm. 3 Esp., (2019)

Luis Carlos Sanchez Lopez; Paola Alexandra Bonifaz Aquino; Maria Monserrate Ponce Ventura; Marcelo Kevin Taiano Espinoza

sinérgico donde aumenta su potencia analgésica, disminuye sus dosis y reduce la posibilidad de aparición de sus efectos secundarios.

\section{Metodología.}

Esta investigación está dirigida al estudio del "Contradicciones de analgesia epidural post cesárea: ¿solución o problema?’. Para realizarlo se usó una metodología tipo descriptiva, con un enfoque documental, es decir, revisar fuentes disponibles en la red, como google académico, con contenido oportuno y relevante desde el punto de vista científico para dar respuesta a lo tratado en el presente artículo y que sirvan de inspiración para realizar otros proyectos. Las mismas pueden ser consultadas al final, en la bibliografía.

\section{Resultados.}

La analgesia post operatoria es una de las áreas más estudiadas y que ocupa al anestesiólogo. Su conocimiento, responsabilidad y dedicación, le perfilan como el profesional idóneo para tratar el dolor mediante el tratamiento más eficiente. La tabla mostrada a continuación resume los tratamientos usados con mayor frecuencia en tratamiento del dolor post cesárea (Marrón, 1993): 
Contradicciones de analgesia epidural post cesárea: ¿solución o problema?

Vol. 3, núm. 3 Esp., (2019)

Luis Carlos Sanchez Lopez; Paola Alexandra Bonifaz Aquino; Maria Monserrate Ponce Ventura; Marcelo Kevin Taiano Espinoza

Tabla Narcóticos epidurales y combinaciones con morfínicos en tratamientos post cesárea

\begin{tabular}{|c|c|c||c|}
\hline \hline Agente & Dosis & Duración & Latencia \\
\hline \hline Morfina & $5 \mathrm{mg}$ & $>10 \mathrm{~h}$ & $45 \mathrm{~min}$ \\
\hline Butorfanol & $1-6 \mathrm{mg}$ & $5-10 \mathrm{~h}$ & \\
\hline \hline Meperidina & $50 \mathrm{mg}$ & $2-3 \mathrm{~h}$ & $15 \mathrm{~min}$ \\
\hline \hline Sufentanyl & $30 \mathrm{ug}$ & $2 \mathrm{~h}$ & $15 \mathrm{~min}$ \\
\hline \hline Fentanyl & $50-100 \mathrm{ug}$ & $1 \mathrm{~h}$ & $15 \mathrm{~min}$ \\
\hline \hline Fentanyl + & $50 \mathrm{ug}$ & & \\
\hline \hline Morfina + & & & \\
\hline
\end{tabular}




\section{Contradicciones de analgesia epidural post cesárea: ¿solución o problema?}

Vol. 3, núm. 3 Esp., (2019)

Luis Carlos Sanchez Lopez; Paola Alexandra Bonifaz Aquino; Maria Monserrate Ponce Ventura; Marcelo Kevin Taiano Espinoza

\begin{tabular}{|l||l|l|l|}
\hline Butorfanol & $4 \mathrm{mg}$ & & \\
& & & \\
\hline
\end{tabular}

Fuente: (Marrón, 1993).

El dolor postoperatorio es una combinación de dos procesos fundamentales, la transmisión de impulsos nocioceptivos aferentes, generados por la lesión tisular y la sensibilización central producida por un estímulo persistente. Los opiáceos medulares pueden alterar ambos procesos mediante la inhibición pre y post sináptica de la excitación neural, reduciendo el estímulo que inicia el proceso de hipersensibilización central (Illodo, 2011). Las ventajas de la acción medular de la morfina en el tratamiento del dolor postoperatorio son ampliamente conocidas. En la medida que la morfina se liga a los receptores no sólo se producirá analgesia; también se producen efectos secundarios, siendo los más frecuentes: prurito, náusea, vómito, depresión respiratoria, retención urinaria, etc.

Dentro de los factores identificables para que una paciente presente mayor riesgo de desarrollar dolor post operación de cesárea están: pacientes con cirugías abdominales previas, operación cesárea anterior, pacientes en tratamiento por dolor crónico, depresión u otros trastornos psicológicos en tratamiento o diagnosticados post parto y la utilización de anestesia general (Domke, 2018). En relación con la calidad de la analgesia post cirugía de operación cesárea, existe una directa relación entre el dolor agudo experimentado y la posibilidad de desarrollar dolor post cesárea. Pacientes que presentan altos índices de dolor en el periodo postoperatorio tienen alta incidencia del dolor crónico post operación cesárea. Dentro de las 


\section{Contradicciones de analgesia epidural post cesárea: ¿solución o problema?}

Vol. 3, núm. 3 Esp., (2019)

Luis Carlos Sanchez Lopez; Paola Alexandra Bonifaz Aquino; Maria Monserrate Ponce Ventura; Marcelo Kevin Taiano Espinoza

estrategias de tratamiento recomendados para prevenir el dolor se encuentran la implementación de analgesia preventiva; es decir la administración de fármacos previo al inicio de la operación.

Estos efectos se presentan generalmente en dosis superiores a las que habitualmente se utilizan, por vía peridural las dosis son menores, las concentraciones plasmáticas disminuyen y por lo tanto, los efectos adversos también. El dolor también puede afectar negativamente a las primeras interacciones entre madre e hijo, en el cuidado del bebé en el puerperio inmediato e incluso puede disminuir la capacidad de una madre a amamantar de manera eficaz. Es necesario que el alivio del dolor sea seguro y efectivo, que no interfiera con la capacidad de la madre para desenvolverse y realizar todo lo concerniente al cuidado de su bebé, y que no produzca efectos adversos en la lactancia.

\section{Opioides en la paciente obstétrica}

La Academia Americana de Pediatría sugiere que los opioides pueden tener cierta compatibilidad con la lactancia, siempre y cuando se administren a dosis bajas, por breves períodos de tiempo y las concentraciones sanguíneas en la madre sean bajas. Los opioides que se pueden utilizar por vía peridural: la morfina y buprenorfina cumplen con las condiciones antes mencionadas, un adecuado nivel de analgesia, presentan mínimos cambios hemodinámicos, sensoriales y efectos adversos.

Los opioides se pueden clasificar según su lipofilicidad. Los opioides más lipofílicos como el sufentanil y el fentanil, optimizan en gran medida la calidad de la anestesia intra operatoria, ya que tienen un inicio de acción más rápido pero una duración más corta, que los que tienen menor lipofilicidad, siendo el tiempo medio de efecto del fentanil neuroaxial 


\section{Contradicciones de analgesia epidural post cesárea: ¿solución o problema?}

Vol. 3, núm. 3 Esp., (2019)

Luis Carlos Sanchez Lopez; Paola Alexandra Bonifaz Aquino; Maria Monserrate Ponce Ventura; Marcelo Kevin Taiano Espinoza

aproximadamente 4 horas. En contraste, los opioides hidrofílicos como la morfina tienen una duración mayor, entre 14 y 36 horas, pero con un inicio de acción más tardío, en comparación con los opioides que tienen mayor lipofilicidad. La dosificación óptima de la morfina intratecal es incierta. La dosis de la morfina intratecal está relacionada con la duración del efecto más que con la eficacia analgésica. Dosis mayores de $100 \mathrm{mg}$ prolongan el tiempo requerido para una analgésica de rescate en promedio 4,5 horas en comparación a dosis menores de 100 mg (Córdova, 2000).

La nalbufina es un opioide sintético con propiedades agonistas y antagonistas que está químicamente emparentado con la naxolona (un narcótico antagonista) y con la oximorfona (un potente analgésico narcótico), que ha demostrado su eficacia en el control del dolor postoperatorio, con un mínimo de efectos colaterales, como antagonista de los receptores MU, lo que produce reversión de la depresión respiratoria causada por morfina o cualquier otro agonista puro y un efecto KAPPA agonista (cuya localización principal es la médula espinal), que ejerce una profunda analgesia, acompañada de sedación y miosis, pero nula o muy limitada depresión respiratoria.

El metabolismo de la nalbufina tiene lugar en el hígado. Excretándose sus metabolitos en la orina, la bilis y las heces. Sus parámetros farmacocinéticas son Vd :2.9 L/kg, depuración 6-22 ml/kg/min, TY ${ }_{\mathrm{i}}$ beta: de 120-210 min. Aunque es mayor la densidad de receptores Kappa en las astas dorsales, su efecto analgésico es mínimo. No tiene efectos adversos sobre el sistema cardiovascular, aun en pacientes con infarto agudo de miocardio a dosis de $0.15 \mathrm{mg} / \mathrm{kg}$, tiene un efecto «tope» (Osorio, 2016). No causa efectos colaterales en neonatos, cuando se utiliza en la analgesia obstétrica En estudios sobre analgesia epidural post cesárea con 20-30 mg de 


\section{Contradicciones de analgesia epidural post cesárea: ¿solución o problema?}

Vol. 3, núm. 3 Esp., (2019)

Luis Carlos Sanchez Lopez; Paola Alexandra Bonifaz Aquino; Maria Monserrate Ponce Ventura; Marcelo Kevin Taiano Espinoza

nalbufina, se proporciona una analgesia efectiva durante $2-4$ h y sólo de 77 minutos con $10 \mathrm{mg}$, si la anestesia quirúrgica se realiza con lidocaína. La nalbufina se une muy poco a las proteínas de plasma y atraviesa la placenta produciendo unas concentraciones iguales o mayores que las observadas en el plasma; por otra parte, se ha demostrado que la nalbufina deprime el centro respiratorio en proporción similar a la morfina; sin embargo, no afecta la depresión al aumentar la dosis.

La morfina y sus congéneres generan efectos terapéuticos sobre cerebro y médula espinal, aunque también actúan en sistema digestivo, producen cambios afectivos, depresión respiratoria, náusea, vómito y cambios en el diámetro pupilar. Afectan también las secreciones gástricas la motilidad intestinal, además de tener efectos endocrinos, urinarios y autónomos; de ahí sus efectos adversos. Tras su administración vía peridural tiene un inicio de acción entre 30 y 40 minutos y una vida media efectiva de hasta $12-24$ horas; la dosis es de $0.1 \mathrm{mg} / \mathrm{kg}$ o estandarizada de 2 a $3 \mathrm{mg}$ por dosis (Templos, 2008). Se ha demostrado en estudios en animales que inclusive la adición de adrenalina a dosis comerciales incrementa los tiempos de vida media en el espacio peridural de ciertos opioides.

La Asociación Americana de Anestesiología recomienda el uso de opioides neuroaxiales en lugar de bolos intermitentes parenterales para la analgesia postoperatoria en la cesárea, pero su uso rutinario suele estar limitado por la disponibilidad del personal necesario para realizar seguimiento los posibles efectos secundarios. La analgesia epidural tiene un mejor control del dolor que la medicación manejada por el paciente mediante el uso de medicina oral, pero es más costosa, por lo que la segunda se considera la alternativa más costo-efectiva (Ramos, 2017). 


\section{Contradicciones de analgesia epidural post cesárea: ¿solución o problema?}

Vol. 3, núm. 3 Esp., (2019)

Luis Carlos Sanchez Lopez; Paola Alexandra Bonifaz Aquino; Maria Monserrate Ponce Ventura; Marcelo Kevin Taiano Espinoza

\section{Analgésicos No-Opioides}

La analgesia multimodal permite conseguir una analgesia de calidad y con menos efectos secundarios gracias al uso de diferentes analgésicos o técnicas analgésicas. El uso de analgesia multimodal es importante para optimizar la calidad de la analgesia postoperatoria y disminuir así el requerimiento de opioides en el periodo postparto. El uso de fármacos antiinflamatorios no esteroidales (AINEs) es un componente clave de la analgesia multimodal. Los AINEs disminuyen entre $30 \%$ a $50 \%$ el requerimiento de opioides, lo que reduce la incidencia de efectos adversos asociados a estos últimos (Tornero, 2017). En pacientes sin contraindicaciones, los AINEs deberían ser administrados de forma rutinaria en el periodo postparto. Existen pocos estudios que evalúen la eficacia de los inhibidores de la ciclooxigenasa 2 para la analgesia post operación cesárea, sugiriendo la evidencia disponible, que tienen eficacia limitada; por lo que debiesen ser reservados para pacientes intolerantes a los AINEs no selectivos.

La analgesia postoperatoria multimodal es la más empleada actualmente, comprende la combinación de varias técnicas y analgésicos, como por ejemplo (González, 2005):

$\checkmark$ Utilización de AINE y técnicas de analgesia regional (bloqueos nerviosos periféricos y de plexos).

$\checkmark$ Opioides vía endovenosa con sistemas de PCA (analgesia controlada por el paciente), la cual puede ser empleada en previo entrenamiento del paciente más AINE y/o bloqueos.

$\checkmark$ Epidurales continúas con bombas de infusión o en bolos más AINE.

$\checkmark$ AINE y opioides e.v. 


\section{Contradicciones de analgesia epidural post cesárea: ¿solución o problema?}

Vol. 3, núm. 3 Esp., (2019)

Luis Carlos Sanchez Lopez; Paola Alexandra Bonifaz Aquino; Maria Monserrate Ponce Ventura; Marcelo Kevin Taiano Espinoza

$\checkmark$ Epidurales donde se combinan anestésicos locales y adyuvantes como opioides, bloqueantes de los receptores N-metil-D-aspartato (NMDA), benzodiacepinas, agonistas alfa 2 adrenérgicos entre otros.

$\checkmark$ Infiltración de campo con anestésicos locales más AINE y/u opioides.

Con la analgesia postoperatoria multimodal se emplean dosis menores debido a que la combinación de técnicas y medicamentos potencia el efecto analgésico, brindando una mejor analgesia postoperatoria con menos efectos colaterales.

En el contexto peri operatorio, el acetaminofeno disminuye el requerimiento de opioides de aproximadamente $20 \%$. Como provee una analgesia efectiva con mínimos efectos adversos y escasa excreción a través de la leche materna, el acetaminofeno es un componente importante de la analgesia multimodal. El uso combinado de AINEs y acetaminofeno tienen un efecto sinérgico. Debido a este sinergismo, es altamente aconsejable que ambas drogas sean utilizadas en conjunto. Es así como, por ejemplo, la administración combinada de diclofenaco y acetaminofeno en pacientes intervenidas de una operación cesárea reduce en $38 \%$ el uso de morfina, en comparación al uso de acetaminofeno como droga única (Tornero, 2017).

Los glucocorticoides tienen propiedades analgésicas, antieméticas y antinflamatorias. Una dosis única de dexametasona preoperatoria reduce el dolor en comparación con el placebo en pacientes con anestesia general, además de disminuir la incidencia de náuseas y vómitos postoperatorios. Se han descrito dosis de entre 1,25 hasta $20 \mathrm{mg}$, sin haberse determinado aún las dosis óptimas. La administración de una dosis única de $0,05 \mathrm{mg} / \mathrm{Kg}$ endovenoso previo a la operación cesárea bajo anestesia espinal fue útil en reducir el dolor posoperatorio en las primeras 


\section{Contradicciones de analgesia epidural post cesárea: ¿solución o problema?}

Vol. 3, núm. 3 Esp., (2019)

Luis Carlos Sanchez Lopez; Paola Alexandra Bonifaz Aquino; Maria Monserrate Ponce Ventura; Marcelo Kevin Taiano Espinoza

16 horas en comparación con pacientes que recibieron placebo (Domke, 2018). Más de la mitad de las pacientes que recibieron dexametasona, presentaron prurito perianal. No existen otros estudios que validen la administración de dexametasona previo a la operación cesárea como útiles en reducir el dolor post quirúrgico.

La gabapentina es una droga anticonvulsivante con propiedades analgésicas significativas. Se une a canales de calcio voltaje-dependientes ubicados en el terminal presináptico de los ganglios de las raíces dorsales y de las neuronas de la asta posterior de la medula espinal, previniendo así la liberación de neurotransmisores excitatorios. Ha sido usado en diversas cirugías, ya sea de forma preventiva o como suplemento analgésico postoperatorio. En pacientes sometidas a operación cesárea existen resultados controversiales. Estudios iniciales han sugerido que una dosis única de $600 \mathrm{mg}$ preoperatorio disminuye el dolor postoperatorio y mejora la satisfacción materna, pero estudios posteriores no han logrado validar dichos resultados. La gabapentina presenta además efectos adversos frecuentes como sedación y mareo, presentando además una alta transferencia placentaria y excreción por la leche materna. Es debido a la poca evidencia científica en relación a su eficacia analgésica, como también, a la preocupación por los efectos adversos maternos y neonatales, que no es aconsejable su uso de forma rutinaria en pacientes sometidas a operación cesárea.

\section{Efectos adversos de la morfina}

La morfina es un alcaloide del opio preparado con sulfato por neutralización con ácido sulfúrico. Esta es una sustancia controlada, opioide agonista utilizada en pre medicación, anestesia, analgesia, tratamiento del dolor asociado a la isquemia miocárdica y para la disnea 


\section{Contradicciones de analgesia epidural post cesárea: ¿solución o problema?}

Vol. 3, núm. 3 Esp., (2019)

Luis Carlos Sanchez Lopez; Paola Alexandra Bonifaz Aquino; Maria Monserrate Ponce Ventura; Marcelo Kevin Taiano Espinoza

asociada al fracaso ventricular izquierdo agudo y edema pulmonar. Puede reducir la presión arterial por inducir histamino liberación, o bradicardia por aumento de la actividad del vago. Existe la posibilidad de que la morfina tenga un efecto directo depresor sobre el nodo sinusal y actuar disminuyendo la conducción por el nodo aurículo ventricular. La morfina produce depresión respiratoria, la dosis depende principalmente de un efecto depresor directo sobre el centro de la respiración en el cerebro. Este se caracteriza por disminución de la respuesta al dióxido de carbono aumento de la $\mathrm{PaCO} 2$ basal y desplazamiento de la curva de respuesta al CO2 a la derecha (Romero, 2019) .

Como efectos positivos la morfina disminuye el flujo sanguíneo cerebral y la presión intracraneal con ventilación controlada. Como efectos negativos puede causar rigidez muscular, especialmente en los músculos abdominales y torácicos, cuando es administrada a grandes dosis. Puede causar espasmo del tracto biliar y aumentar las presiones del conducto biliar común que puede asociarse a distress epigástrico o cólico biliar. El estreñimiento puede acompañar a la terapia con morfina secundaria a la reducción de las contracciones peristálticas de los intestinos y aumento del tono del esfínter pilórico, válvula ileocecal y esfínter anal. La morfina puede causar vómitos y náuseas por estimulación directa de la zona "trigger" quimiorreceptora en el suelo del cuarto ventrículo, por aumento de las secreciones gastrointestinales y disminución de la propulsión intestinal (Romero, 2019).

La buprenorfina es un opiáceo agonista-antagonista o agonista parcial. Su potencia analgésica es 33 veces superior a la morfina. Ejerce su efecto analgésico por interacción parcial con el receptor mu, aunque también se une a receptores delta y kappa. La duración de la analgesia es superior a 10 horas; tiene metabolismo hepático y su excreción es renal; su volumen 


\section{Contradicciones de analgesia epidural post cesárea: ¿solución o problema?}

Vol. 3, núm. 3 Esp., (2019)

Luis Carlos Sanchez Lopez; Paola Alexandra Bonifaz Aquino; Maria Monserrate Ponce Ventura; Marcelo Kevin Taiano Espinoza

de distribución es de $2.8 \mathrm{~L} / \mathrm{kg}$ y su aclaramiento es de $20 \mathrm{ml} / \mathrm{kg} / \mathrm{min}$. Las dosis analgésicas iniciales que se recomiendan son de 0.3 a $0.4 \mathrm{mg}$ (Osorio, 2016). Por sus características de liposolubilidad y duración intermedia -principalmente, en los receptores espinales-, permite su uso con grandes perspectivas en dolor posoperatorio. Es de metabolismo hepático y sus metabolitos son eliminados por vía biliar.

De inicio de acción lento, su duración analgésica vía intravenosa está considerada por más de 6 h y por vía sub aracnoidea y peridural la cinética se modifica alargando los tiempos de vida media, la dosis promedio vía peridural de $3 \mu \mathrm{g} / \mathrm{kg}$. La buprenorfina tiene efectos farmacológicos principalmente en el sistema nervioso central y gastrointestinal La analgesia, que es el efecto clásico y el más estudiado, se produce por acción a nivel supra espinal y espinal, inhibiendo la transmisión del dolor y los componentes emocional y afectivo asociados. La estimulación de receptores "p" ubicados en las vías del dolor produce analgesia de mayor potencia, está considerada para el tratamiento de dolor moderado a severo, postoperatorio.

Los reportes de estudios con morfina peridural como dosis única mencionan entre 12 y 24 h de duración de analgesia con un mínimo de efectos adversos, siendo el prurito uno de los más frecuentes. El prurito es efectivamente el efecto adverso que más se presentó en las fuentes consultadas, siendo leve en todos los casos y sin llegar a requerir de la administración de difenhidramina. La analgesia es efectiva pero no suficiente con una dosis única, siendo una buena opción la administración de dosis mínimas (2 a 3 mg vía peridural de morfina) pero subsecuentes para lograr una buena calidad es el control del dolor con pocos o nulos efectos indeseables. Otro efecto deseable de este opioide es el costo. Aproximadamente una dosis de 2 mg tiene un costo de entre 20 y 25 pesos, para una duración de entre 8 y 12 h en comparación 


\section{Contradicciones de analgesia epidural post cesárea: ¿solución o problema?}

Vol. 3, núm. 3 Esp., (2019)

Luis Carlos Sanchez Lopez; Paola Alexandra Bonifaz Aquino; Maria Monserrate Ponce Ventura; Marcelo Kevin Taiano Espinoza

con el ketorolaco que tiene un costo comercial aproximadamente de 40 pesos una dosis de $30 \mathrm{mg}$ y en promedio se llegan a utilizar $60 \mathrm{mg}$ cada $8 \mathrm{~h}$ con un menor (hay que recordar que la morfina vía peridural potencializa hasta 10 veces su efecto) (Illodo, 2011), por lo que se considera una excelente opción por su duración y sus mínimos efectos adversos, además de que está aprobada por revisiones internacionales de fármacos y lactancia para su administración, por no presentar metabolitos en leche materna por las dosis mínimas administradas, por el período de tiempo y por la vía de administración que utilizamos en nuestro estudio.

\section{Contraindicaciones}

Las pacientes con lesiones cardiacas obstructivas serias y compromiso hemodinámico, tales como: estenosis aórtica, estenosis mitral, hipertensión pulmonar y coartación aórtica, no son candidatas para la aplicación de ésta técnica por no tolerar los cambios rápidos a nivel de la pre y post carga. Los casos de Shunt cardíaco de derecha a izquierda puede incrementar se por la caída de la resistencia vascular sistémica. En antecedentes previos de esclerosis múltiple, puede haber re activación de la enfermedad por lo que debe ser cuidadosamente considerada. Son contraindicaciones absolutas para realizar técnicas conductivas, (peridural y raquídea): los trastornos de la coagulación, la hipovolemia severa, la hipotensión, la infección en el sitio de punción, y los casos de hipertensión endocraneana.

\section{Complicaciones}

La cefalea post-punción raquídea, o secundaria a raquídea accidental de la duramadre en la punción peridural, aparece usualmente a las cuarenta y ocho horas, típicamente es frontooccipital, irradiada a cuello y hombro, agravada por la posición sentada y acompañada por 


\section{Contradicciones de analgesia epidural post cesárea: ¿solución o problema?}

Vol. 3, núm. 3 Esp., (2019)

Luis Carlos Sanchez Lopez; Paola Alexandra Bonifaz Aquino; Maria Monserrate Ponce Ventura; Marcelo Kevin Taiano Espinoza

otros síntomas como fotofobia, vómito, tinitus y otros. Otros efectos descritos han sido isquemia medular, por hipotensión prolongada, posición in- correcta o por compresión, bradicardia, bloqueos cardiacos y alteraciones del ritmo cardíaco, asociado con los anestésico s locales y relacionado con ambas técnicas (Veléz, 1998).

\section{Conclusiones.}

Los pacientes que presentan altos índices de dolor en el periodo postoperatorio tienen alta incidencia del dolor crónico post operación cesárea. Dentro de las estrategias de tratamiento recomendados para prevenir el dolor se encuentran la implementación de analgesia preventiva; es decir la administración de fármacos previo al inicio de la operación. Estos efectos perjudiciales para la salud se presentan generalmente en dosis superiores a las que habitualmente se utilizan, por vía peridural las dosis son menores, las concentraciones plasmáticas disminuyen y por lo tanto los efectos adversos también.

La dosificación óptima de la morfina intratecal es incierta. La dosis de la morfina intratecal está relacionada con la duración del efecto más que con la eficacia analgésica. La nalbufina es un opioide sintético con propiedades agonistas y antagonistas que ha demostrado su eficacia en el control del dolor postoperatorio, con un mínimo de efectos colaterales, como antagonista de los receptores MU, lo que produce reversión de la depresión respiratoria causada por morfina o cualquier otro agonista puro que ejerce una profunda analgesia, acompañada de sedación y miosis, pero nula o muy limitada depresión respiratoria.

La analgesia multimodal permite conseguir una analgesia de calidad y con menos efectos secundarios gracias al uso de diferentes analgésicos o técnicas analgésicas. El uso de analgesia 


\section{Contradicciones de analgesia epidural post cesárea: ¿solución o problema?}

Vol. 3, núm. 3 Esp., (2019)

Luis Carlos Sanchez Lopez; Paola Alexandra Bonifaz Aquino; Maria Monserrate Ponce Ventura; Marcelo Kevin Taiano Espinoza

multimodal es importante para optimizar la calidad de la analgesia postoperatoria y disminuir así el requerimiento de opioides en el periodo postparto. Una analgesia efectiva con mínimos efectos adversos y escasa excreción a través de la leche materna, el acetaminofeno es un componente importante de la analgesia multimodal. El uso combinado de AINEs y acetaminofeno tienen un efecto sinérgico. Debido a este sinergismo, es altamente aconsejable que ambas drogas sean utilizadas en conjunto.

Por todo lo anterior podemos concluir que la analgesia en obstetricia es controversial y por ese motivo debe encontrarse una línea de manejo que resulte con el mínimo de efectos adversos para la madre y el producto con una buena calidad en el manejo del dolor. La ciencia ha avanzado, pero todavía no existe la fórmula única e inequívoca.

\section{Bibliografía.}

Córdova, A. (2000). Analgesia post cesárea con sulfato de morfina en infución epidural. Revista Mexicana de Anestesiología , 23(1), 20-24.

Domke, R. (2018). Manejo del dolor agudo postoperatorio en operación de cesárea. Revista Chilena Obstetricia y Ginecología , 83(6), 635-642.

González, N. (2005). Analgesia multimodal postoperatoria. Revista de la Sociedad Española del Dolor Vol, 12(2), 112-118.

Illodo, M. D. (2011). Análisis de la eficacia y seguridad de la administración de cloruro mórfico epidural para el dolor postoperatorio tras cesárea. Revista Soc.iedad Española del Dolor, 18(1), 6-11.

Marrón, G. (1993). Narcóticos epidurales en anestesia obstétrica. Revista Mexicana de Anestesiología, 16, 31-37.

Osorio, A. (Mayo de 2016). Nalbufina versus Buprenorfina peridural en el tratamiento del dolor. Obtenido de Universidad Veracruzana: www.cdigital.uv.mx

Ramos, G. (2017). Manejo analgésico durante el postoperatorio de cesárea: estrategias farmacológicas. Revista Colombiana de Anestesiología, 45(4), 327-334. 


\section{Contradicciones de analgesia epidural post cesárea: ¿solución o problema?}

Vol. 3, núm. 3 Esp., (2019)

Luis Carlos Sanchez Lopez; Paola Alexandra Bonifaz Aquino; Maria Monserrate Ponce Ventura; Marcelo Kevin Taiano Espinoza

Romero, M. (2019). Contraindicaciones de analgesia epidural con morfina post cesárea. Revista Científica Mundo de la Investigación y el Conocimiento, 3(3), 596-613.

Templos, L. (2008). Comparación entre buprenorfina y morfina peridural para manejo de dolor postoperatorio en paciente sometida a cesárea. Revista Mejicana de Anestesiología, 31(3), 172-178.

Tornero, C. (2017). Analgesia multimodal y anestesia regional. Revista Española de Anestesiología y Reanimación, 64(7), 401-405.

Veléz, P. (1998). Alternativas anestésicas para cesárea. Revista Colombiana de Anestesiología, 25(73), 73-80 .

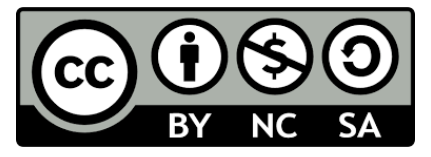

RECONOCIMIENTO-NOCOMERCIAL-COMPARTIRIGUAL

CC BY-NC-SA

ESTA LICENCIA PERMITE A OTROS ENTREMEZCLAR, AJUSTAR Y CONSTRUIR A PARTIR DE SU OBRA CON FINES NO COMERCIALES, SIEMPRE Y CUANDO LE RECONOZCAN LA AUTORÍA Y SUS NUEVAS CREACIONES ESTÉN BAJO UNA LICENCIA CON LOS MISMOS TÉRMINOS. 